\title{
Theoretical and Experimental Studies on the
}

\section{Controllable Pancake Bouncing Behavior of}

\section{Droplets}

Huaping $W u^{* \dagger}$, Kunpeng Jiang ${ }^{\dagger}$, Zhenxiong Xü , Sihang Yu $u^{\dagger}$ Xiang Peng ${ }^{\dagger}$, Zheng Zhang ${ }^{\dagger}$, Hao Bai ${ }^{\ddagger}$, Aiping Liu ${ }^{\S}$, Guozhong Chai*†

$\dagger$ Key Laboratory of E\&M, Zhejiang University of Technology, Ministry of Education \& Zhejiang Province, Hangzhou 310014, China

†Center for Optoelectronics Materials and Devices, Zhejiang Sci-Tech University, Hangzhou 310018, China

\section{Supporting Videos:}

Video S1: The rebound of a droplet on the SCAS with $S / D_{\mathrm{b}}=0.5$.

Video S2: The rebound of a droplet on the SCAS with $S / D_{\mathrm{b}}=1.0$.

Video S3: The rebound of a droplet on the SCAS with $S / D_{\mathrm{b}}=1.5$.

Video S4: The rebound of a droplet on the SCAS with $S / D_{\mathrm{b}}=2.0$.

Video S5: The rebound of a droplet on the SCAS with $S / D_{\mathrm{b}}=2.5$. 


\section{C1. Wetting states of a droplet on the SCAS.}

Figure 1 is the schematic of a droplet on the superhydrophobic conical array surface (SCAS). From the following geometric relationships

$$
\begin{aligned}
& S_{\text {ext }}=2 \pi R(\theta)^{2}(1-\cos \theta), \\
& S_{\text {base }}=\pi R(\theta)^{2} \sin ^{2} \theta, \\
& \alpha=\arctan \left(\frac{D_{\mathrm{b}}-D_{\mathrm{t}}}{2 H}\right),
\end{aligned}
$$

the radius of the droplet $R(\theta)$ related to the apparent contact angle $\theta$ can be expressed as

$$
R(\theta)=\left(\frac{3 V}{\pi} \frac{1}{2-3 \cos \theta+\cos ^{3} \theta}\right)^{1 / 3} .
$$

For the above system, the free energy includes the energy of interface free energy, potential energy and line tension. It is assumed that the influence of gravity and line tension can be ignored, and the total free energy of the system $G$ is taken as

$$
G=\gamma_{\mathrm{LV}} S_{\mathrm{LV}}+\gamma_{\mathrm{SL}} S_{\mathrm{SL}}+\gamma_{\mathrm{SV}} S_{\mathrm{SV}},
$$

where $S_{\mathrm{LV}}, S_{\mathrm{SL}}$ and $S_{\mathrm{SV}}$ are the liquid-air interface, the solid-liquid interface and the solid-gas interface, respectively, and $\gamma_{\mathrm{LV}}, \gamma_{\mathrm{SL}}$ and $\gamma_{\mathrm{SV}}$ are the corresponding surface energies of the droplet.

According to the geometry, the area fraction $f$, the infiltration area fraction $f^{\prime}$ and the roughness factor $r$ of the conical microstructures and the spherical microstructures on the SCAS are listed in Table S1. 
Table S1. The infiltration area fraction and roughness factor of the conical microstructures and the spherical microstructures on the SCAS.

\begin{tabular}{lccc}
\hline & Area fraction & Infiltration area fraction & Roughness factor \\
\hline $\begin{array}{c}\text { Conical } \\
\text { micro- } \\
\text { structures }\end{array}$ & $f_{1}=\frac{\pi\left(\frac{D_{\mathrm{t}}}{2}+x \tan \alpha\right)^{2}}{\left(S+D_{\mathrm{b}}\right)^{2}}$ & $f_{1}^{\prime}=\frac{\pi\left(\frac{D_{\mathrm{t}}}{2}\right)^{2}+\pi\left(D_{\mathrm{t}}+x \tan \alpha\right) \frac{x}{\cos \alpha}}{\left(S+D_{\mathrm{b}}\right)^{2}}$ & $r_{1}=\frac{\left(S+D_{\mathrm{b}}\right)^{2}-\pi\left(\frac{D_{\mathrm{b}}}{2}\right)^{2}+\pi\left(\frac{D_{\mathrm{t}}}{2}\right)^{2}+\pi\left(\frac{D_{\mathrm{t}}+D_{\mathrm{b}}}{2}\right) \frac{H}{\cos \alpha}}{\left(S+D_{\mathrm{b}}\right)^{2}}$ \\
$\begin{array}{c}\text { Spherical } \\
\text { micro- } \\
\text { structures }\end{array}$ & $f_{2}=\frac{\pi\left(a x_{2}-x_{2}^{2}\right)}{(a+b)^{2}}$ & $f_{2}^{\prime}=\frac{\pi a^{2}}{(a+b)^{2}} \frac{x_{2}}{a}$ & $r_{2}=1+\frac{4 \pi(0.5 a)^{2}}{(a+b)^{2}}$ \\
\hline
\end{tabular}

(1) Assuming that both the conical microstructures and the spherical microstructures are in the Composite wetting state $\left(0 \leq x_{1}<H, 0 \leq x_{2}<a\right)$, the total free energy of the system is

$$
\begin{aligned}
G_{\mathrm{COMP}-\mathrm{COMP}} & =\gamma_{\mathrm{LV}}\left[S_{\text {ext }}+S_{\text {base }}\left(1-f_{1}\right) y_{1}+S_{\text {base }} f_{1}^{\prime}\left(1-f_{2}\right) y_{2}\right]+\gamma_{\mathrm{SL}}\left(S_{\text {base }} f_{1}^{\prime} f_{2}^{\prime}\right) \\
& +\gamma_{\mathrm{SV}}\left[\left(S_{\text {total }}-S_{\text {base }}\right) r_{1} r_{2}+S_{\text {base }}\left(r_{1}-f_{1}^{\prime}\right) r_{2}+S_{\text {base }} f_{1}^{\prime}\left(r_{2}-f_{2}^{\prime}\right)\right], \\
& =\gamma_{\mathrm{LV}} S_{\text {ext }}+\gamma_{\mathrm{SV}} S_{\text {total }} r_{1} r_{2}+\gamma_{\mathrm{LV}} S_{\text {base }} C_{1}
\end{aligned}
$$

where

$$
C_{1}=-\left[f_{1}^{\prime} f_{2}^{\prime} \cos \theta_{0}+\left(f_{1}-1\right) y_{1}+f_{1}^{\prime}\left(f_{2}-1\right) y_{2}\right]
$$

(2) Assuming that the spherical microstructures are in the Composite wetting state, the conical microstructures are in the Wenzel state $\left(x_{1}=H, 0 \leq x_{2}<a\right)$, the total free energy of the system is 


$$
\begin{aligned}
G_{\mathrm{COMP}-\mathrm{W}} & =\gamma_{\mathrm{LV}}\left[S_{\text {ext }}+S_{\text {base }} r_{1}\left(1-f_{2}\right) y_{2}\right]+\gamma_{\mathrm{SL}}\left(S_{\text {base }} r_{1} f_{2}^{\prime}\right) \\
& +\gamma_{\mathrm{SV}}\left[\left(S_{\text {total }}-S_{\text {base }}\right) r_{1} r_{2}+S_{\text {base }} r_{1}\left(r_{2}-f_{2}^{\prime}\right)\right], \\
& =\gamma_{\mathrm{LV}} S_{\text {ext }}+\gamma_{\mathrm{SV}} S_{\text {total }} r_{1} r_{2}+\gamma_{\mathrm{LV}} S_{\text {base }} C_{2}
\end{aligned}
$$

where

$$
C_{2}=-\left[r_{1} f_{2}^{\prime} \cos \theta_{0}+r_{1}\left(f_{2}-1\right) y_{2}\right]
$$

(3) Assuming that the conical microstructures are in the Composite wetting state and the spherical microstructures are in the Wenzel state $\left(0 \leq x_{1}<H, x_{2}=a\right)$, the total free energy of the system is

$$
\begin{aligned}
G_{\mathrm{W}-\mathrm{COMP}} & =\gamma_{\mathrm{LV}}\left[S_{\text {ext }}+S_{\text {base }}\left(1-f_{1}\right) y_{1}\right]+\gamma_{\mathrm{SL}} S_{\text {base }} f_{1}^{\prime} r_{2} \\
& +\gamma_{\mathrm{SV}}\left[\left(S_{\text {total }}-S_{\text {base }}\right) r_{1} r_{2}+S_{\text {base }} r_{2}\left(1-f_{1}^{\prime}\right)\right], \\
& =\gamma_{\mathrm{LV}} S_{\text {ext }}+\gamma_{\mathrm{SV}} S_{\text {total }} r_{1} r_{2}+\gamma_{\mathrm{LV}} S_{\text {base }} C_{3}
\end{aligned}
$$

where

$$
C_{3}=-\left[f_{1}^{\prime} r_{2} \cos \theta_{0}+\left(f_{1}-1\right) y_{2}\right]
$$

(4) Assuming that both the conical microstructures and the spherical microstructures are both in the Wenzel state $\left(x_{1}=H, x_{2}=a\right)$, the total free energy of the system is

$$
\begin{aligned}
G_{\mathrm{W}-\mathrm{W}} & =\gamma_{\mathrm{LV}} S_{\mathrm{ext}}+\gamma_{\mathrm{SL}} S_{\text {base }} r_{1} r_{2}+\gamma_{\mathrm{SV}}\left[\left(S_{\text {total }}-S_{\text {base }}\right) r_{1} r_{2}\right], \\
& =\gamma_{\mathrm{LV}} S_{\mathrm{ext}}+\gamma_{\mathrm{SV}} S_{\text {total }} r_{1} r_{2}+\gamma_{\mathrm{LV}} S_{\text {base }} C_{4}
\end{aligned},
$$

where 


$$
C_{4}=-r_{1} r_{2} \cos \theta_{0} .
$$

Bring Equations (S1) and (S2) into the above formulas (S6, S8, S10, S12),

$$
G=\pi\left(\frac{3 V}{\pi}\right)^{2 / 3} \frac{\left[2 \gamma_{\mathrm{LV}}(1-\cos \theta)+C \gamma_{\mathrm{LV}} \sin ^{2} \theta\right]}{\left(2-3 \cos \theta+\cos ^{3} \theta\right)^{2 / 3}}+\gamma_{\mathrm{SV}} S_{\text {total }} r_{1} r_{2} .
$$

The non-quantized expression of free energy $G^{*}$ obtained from Equation (S14) is

$$
G^{*}=\frac{G-\gamma_{\mathrm{SV}} S_{\text {total }} \gamma_{1} r_{2}}{\gamma_{\mathrm{LV}} \pi^{1 / 3}(3 V)^{2 / 3}}=\frac{2(1-\cos \theta)+C \sin ^{2} \theta}{\left(2-3 \cos \theta+\cos ^{3} \theta\right)^{2 / 3}} .
$$

Solving Equation $\mathrm{S} 15$ gives $\cos \theta=-C$ or $\cos \theta=-1$. When $\cos \theta=-1$, it indicates that the droplet contact angle is $180^{\circ}$ and the droplet does not contact the substrate. It does not conform to the model and therefore is discarded.

When both the conical microstructures and the spherical microstructures are in a composite wetting state $\left(0 \leq x_{1}<H, 0 \leq x_{2}<a\right)$, the minimum energy is obtained as $\cos \theta_{\text {COMP-COMP }}=-C_{1}$. The apparent contact angle equation in this state is

$$
\cos \theta_{\text {СомР-СомР }}=f_{1}^{\prime} f_{2}^{\prime} \cos \theta_{0}+\left(f_{1}-1\right) y_{1}+f_{1}^{\prime}\left(f_{2}-1\right) y_{2} .
$$

Take the second derivative of this with respect to $x_{1}$,

$$
\frac{\partial^{2} \cos \theta_{\text {COMP-COMP }}}{\partial x^{2}}=\frac{2 f_{2}^{\prime} \pi \cos \theta_{0} \frac{\tan \alpha}{\cos \alpha}+2 \pi\left(f_{2}-1\right) \frac{\tan \alpha}{\cos \alpha}+2 \pi(\tan \alpha)^{2}}{\left(S+D_{\mathrm{b}}\right)^{2}} .
$$


Assume that $f_{2} \approx f_{2}^{\prime}$, and the material intrinsic contact angle is $110^{\circ}$, in order that Equation (S17) is greater than 0 , then $\sin \alpha>1-f_{2} / 2$, and the derivative of the Equation (S16) is also greater than 0 . It can be seen that when the half apex angle $\alpha>\arcsin \left(1-f_{2} / 2\right)$, the droplets will infiltrate in the microstructure, and when $f_{2}=0.227$, then $\alpha>62.44^{\circ}$. It can be seen that in this model, the droplets do not infiltrate the conical arrays. From the above Equation (S16), as the $y$ increases, the free energy increases. Therefore, when $y_{1}=y_{2}=1$, the energy is the smallest in this state. That is, the droplet is in the CB-CB state, and the equation for the apparent contact angle of the state is

$$
\cos \theta_{\text {CB-СB }}=\frac{\pi\left(D_{t} / 2\right)^{2}}{\left(S+D_{b}\right)^{2}} \frac{\pi a^{2}}{3(a+b)^{2}} \cos \theta_{0}+\frac{\pi\left(D_{t} / 2\right)^{2}}{\left(S+D_{b}\right)^{2}} \frac{2 \pi a^{2}}{9(a+b)^{2}}-1 .
$$

That is, in the Cassie-Baxter state, the depth of droplet penetration for the conical arrays is $x_{1}=0$, and the spherical structure $x_{2}=0.33 \mathrm{a}$. Similarly, for the other three wetting states, the contact angle equations are

$$
\begin{aligned}
& \cos \theta_{\mathrm{CB}-\mathrm{W}}= {\left[\frac{\left(S+D_{\mathrm{b}}\right)^{2}-\pi\left(\frac{D_{\mathrm{b}}}{2}\right)^{2}+\pi\left(\frac{D_{\mathrm{t}}}{2}\right)^{2}+\pi\left(\frac{D_{\mathrm{t}}+D_{\mathrm{b}}}{2}\right) \frac{H}{\cos \alpha}}{\left(S+D_{\mathrm{b}}\right)^{2}}\right] \times } \\
& {\left[\frac{\pi a^{2}}{3(a+b)^{2}} \cos \theta_{0}+\frac{2 \pi a^{2}}{9(a+b)^{2}}-1\right], } \\
& \cos \theta_{\mathrm{W}-\mathrm{CB}}=\frac{\pi\left(D_{t} / 2\right)^{2}}{\left(S+D_{\mathrm{b}}\right)^{2}}\left(1+\frac{\pi a^{2}}{(a+b)^{2}}\right) \cos \theta_{0}+\frac{\pi\left(D_{\mathrm{t}} / 2\right)^{2}}{\left(S+D_{\mathrm{b}}\right)^{2}}-1,
\end{aligned}
$$




$$
\cos \theta_{\mathrm{w}-\mathrm{w}}=\frac{\left(S+D_{\mathrm{b}}\right)^{2}-\pi\left(\frac{D_{\mathrm{b}}}{2}\right)^{2}+\pi\left(\frac{D_{\mathrm{t}}}{2}\right)^{2}+\pi\left(\frac{D_{\mathrm{t}}+D_{\mathrm{b}}}{2}\right) \frac{H}{\cos \alpha}}{\left(S+D_{\mathrm{b}}\right)^{2}}\left(1+\frac{\pi a^{2}}{(a+b)^{2}}\right) \cos \theta_{0} \cdot(\mathrm{S} 21)
$$
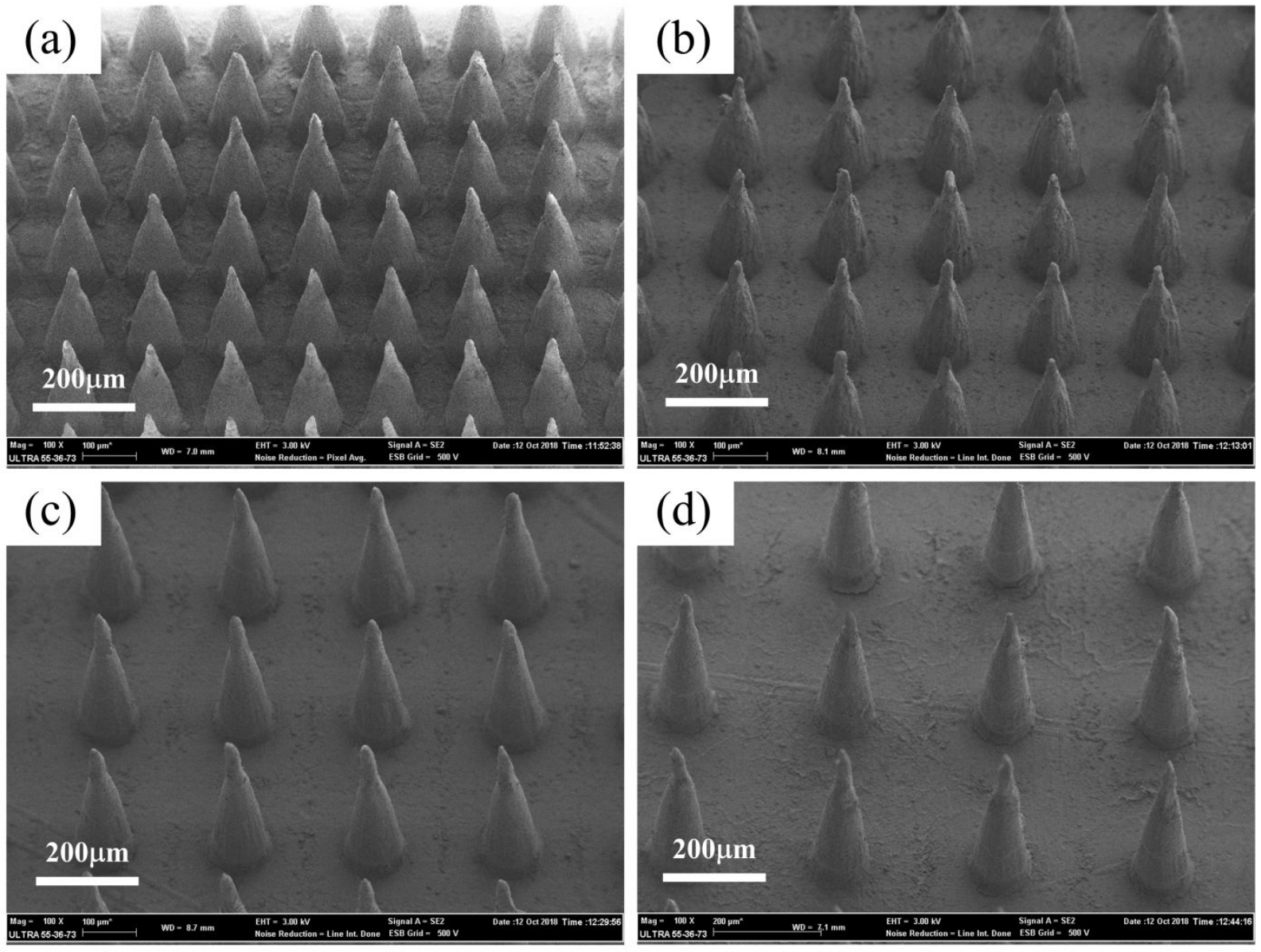

(e)

(f)

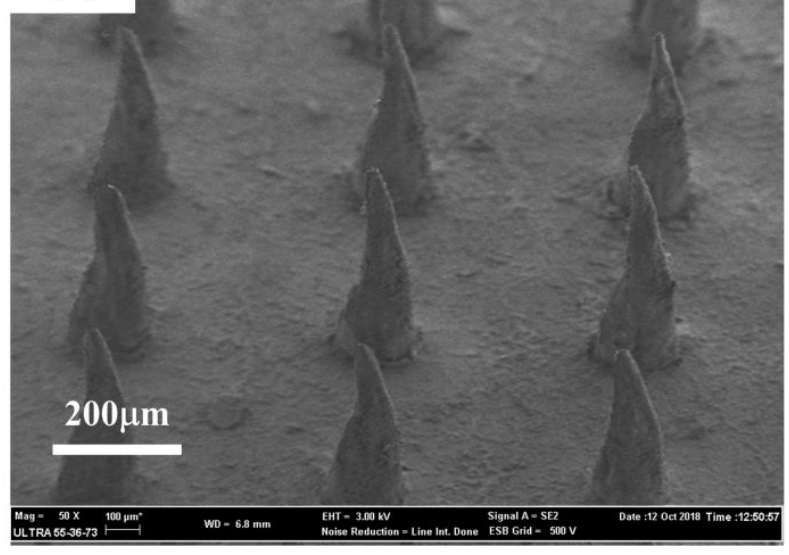

$200 \mu \mathrm{m}$

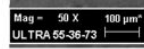

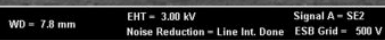

Date: :12 0ct 2018 Time: 12:54:26 
Figure S1. The scanning electron microscopy images of SCAS with different microstructural spacing. The ratio of conical arrays is (a) $S / D_{\mathrm{b}}=0.5$, (b) $S / D_{\mathrm{b}}=1.0$, (c) $S / D_{\mathrm{b}}=1.5$, (d) $S / D_{\mathrm{b}}=2.0$, (e) $S / D_{\mathrm{b}}=2.5$, and (f) $S / D_{\mathrm{b}}=3.0$.

Table S2. Geometrical parameters of the SCAS with different ratios of conical arrays.

\begin{tabular}{ccccc}
\hline$S / D_{\mathrm{b}}$ & $\begin{array}{c}\text { Height } H \\
(\mu \mathrm{m})\end{array}$ & $\begin{array}{c}\text { Top surface diameter } \\
D_{\mathrm{t}}(\mu \mathrm{m})\end{array}$ & $\begin{array}{c}\text { Bottom surface diameter } \\
D_{\mathrm{b}}(\mu \mathrm{m})\end{array}$ & $\begin{array}{c}\text { Bottom spacing between } \\
\text { the two posts } S(\mu \mathrm{m})\end{array}$ \\
\hline 0.5 & 600 & 10 & 100 & 50 \\
1.0 & 600 & 10 & 100 & 100 \\
1.5 & 600 & 10 & 100 & 200 \\
2.0 & 600 & 10 & 100 & 250 \\
2.5 & 600 & 10 & 100 & 300 \\
3.0 & 600 & 10 & 100 & \\
\hline
\end{tabular}

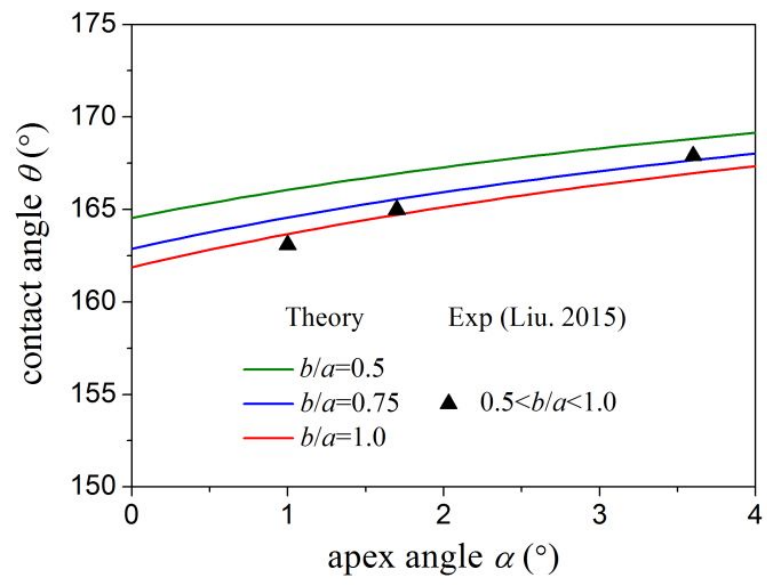

Figure S2. The wetting states of a droplet on the SCAS with different half-apex angles $\left(S / D_{\mathrm{b}}=1\right)$. The solid triangle in the figure is the experimental value of previous experiments. 


\section{C2. The bouncing states of a droplet on the SCAS with different spacing $S / D_{\mathrm{b}}$.}

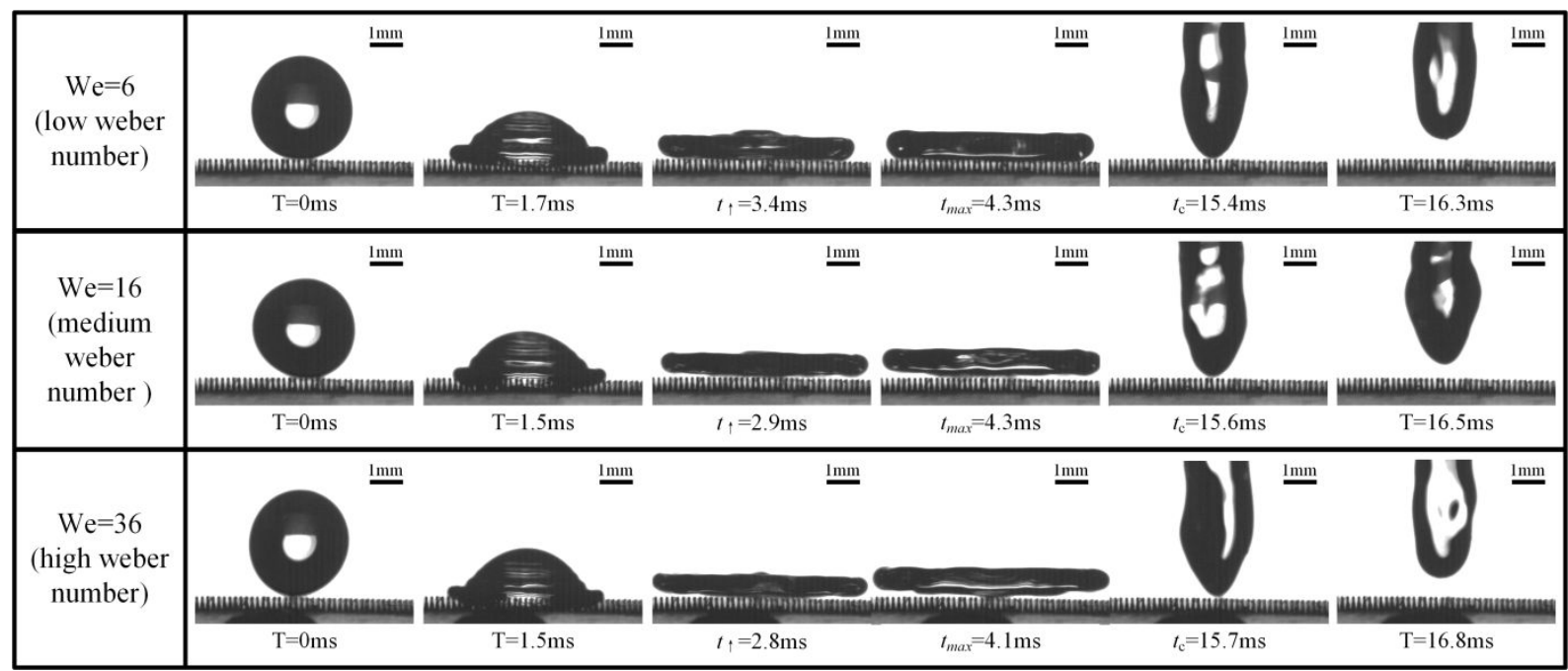

Figure S3-1. Under a different Weber number $W e$, the bouncing states of a droplet on the SCAS with microstructural spacing ratio $S / D_{\mathrm{b}}=0.5$. The droplet radius is $R_{0}=1.45 \mathrm{~mm}$.

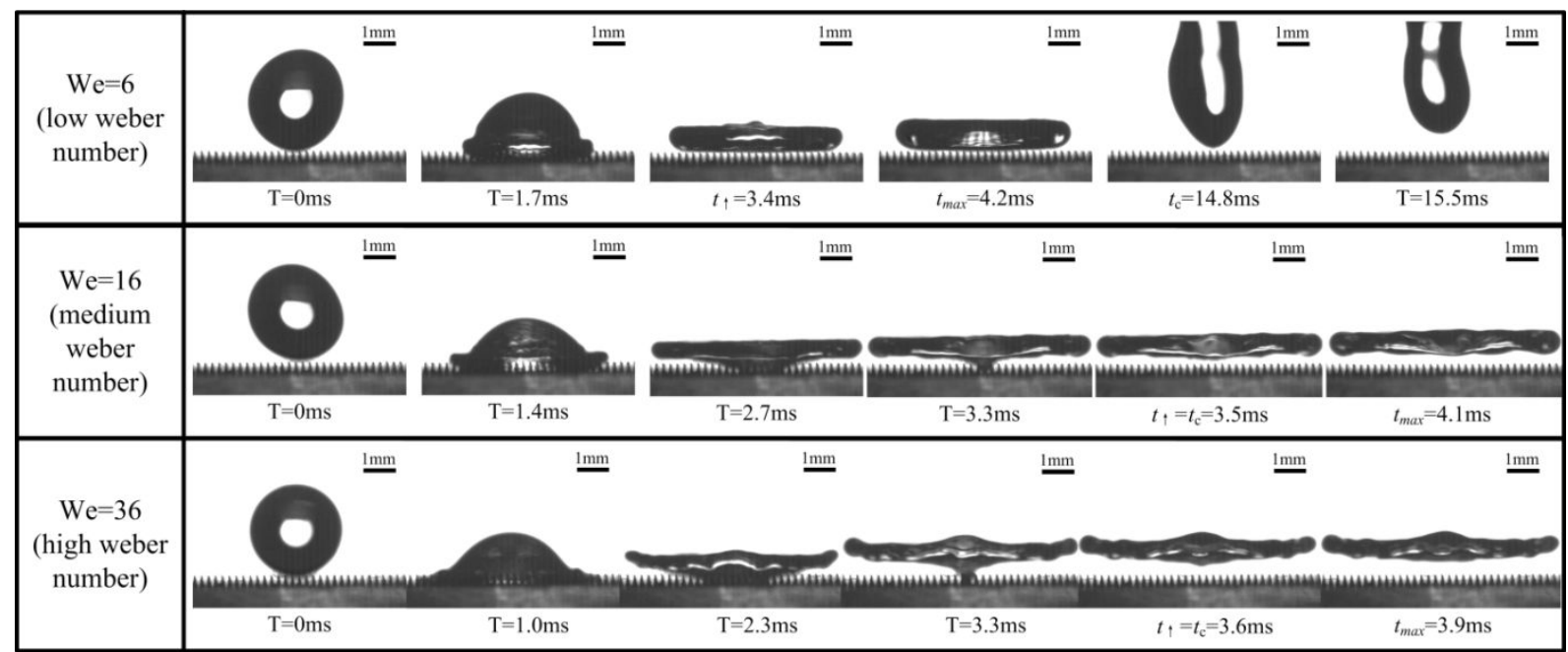

Figure S3-2. Under different Weber number $W e$, the bouncing states of a droplet on the SCAS with microstructural spacing ratio $S / D_{\mathrm{b}}=1.0$. The droplet radius is $R_{0}=1.45 \mathrm{~mm}$. 


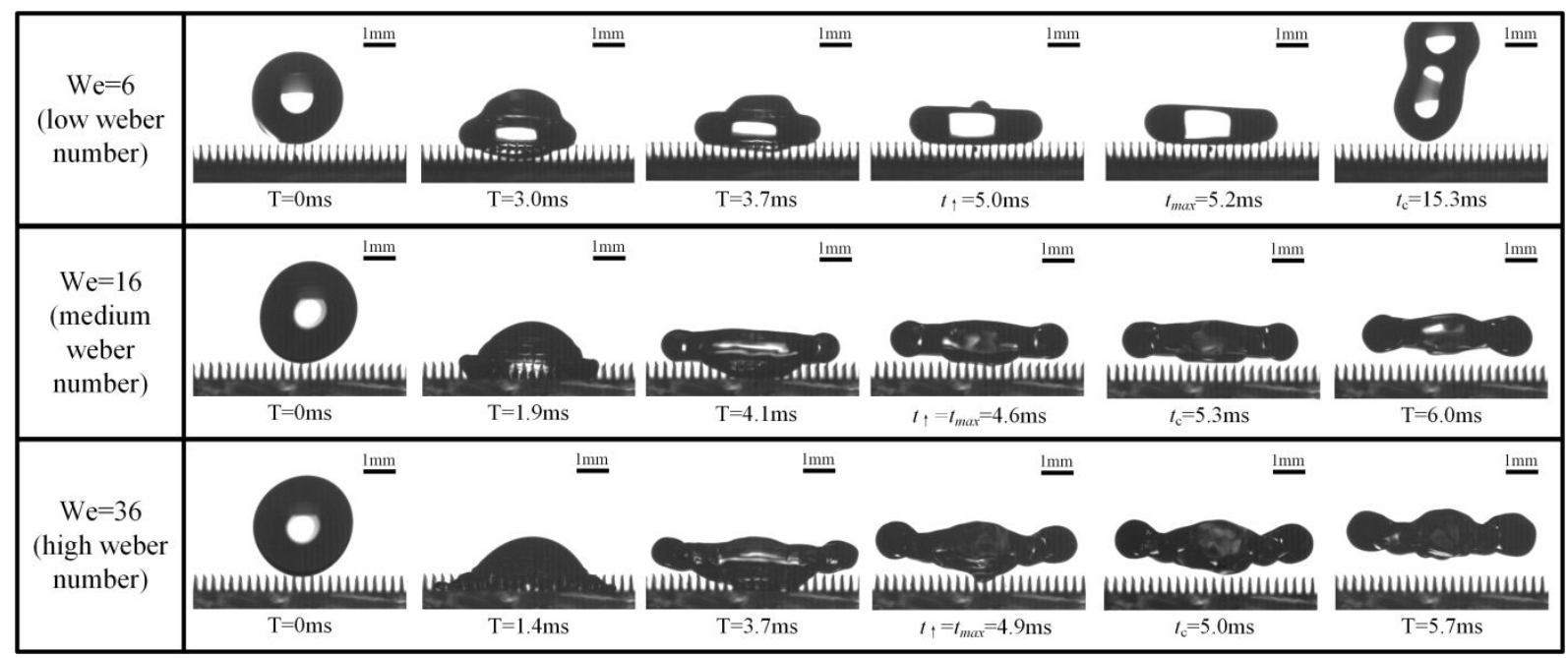

Figure S3-3. Under different Weber number $W e$, the bouncing states of a droplet on the SCAS with microstructural spacing ratio $S / D_{\mathrm{b}}=1.5$. The droplet radius is $R_{0}=1.45 \mathrm{~mm}$.

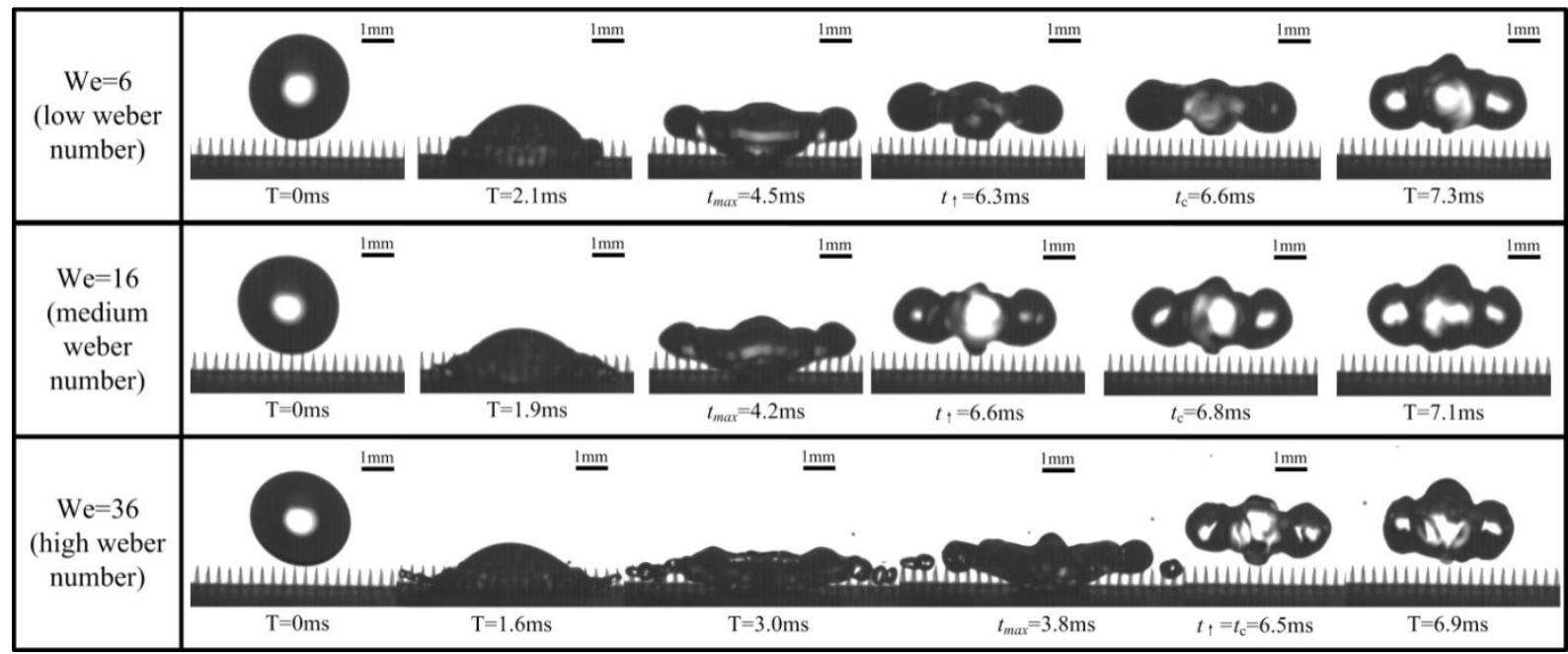

Figure S3-4. Under different Weber number $W e$, the bouncing states of a droplet on the SCAS with microstructural spacing ratio $S / D_{\mathrm{b}}=2.0$. The droplet radius is $R_{0}=1.45 \mathrm{~mm}$. 


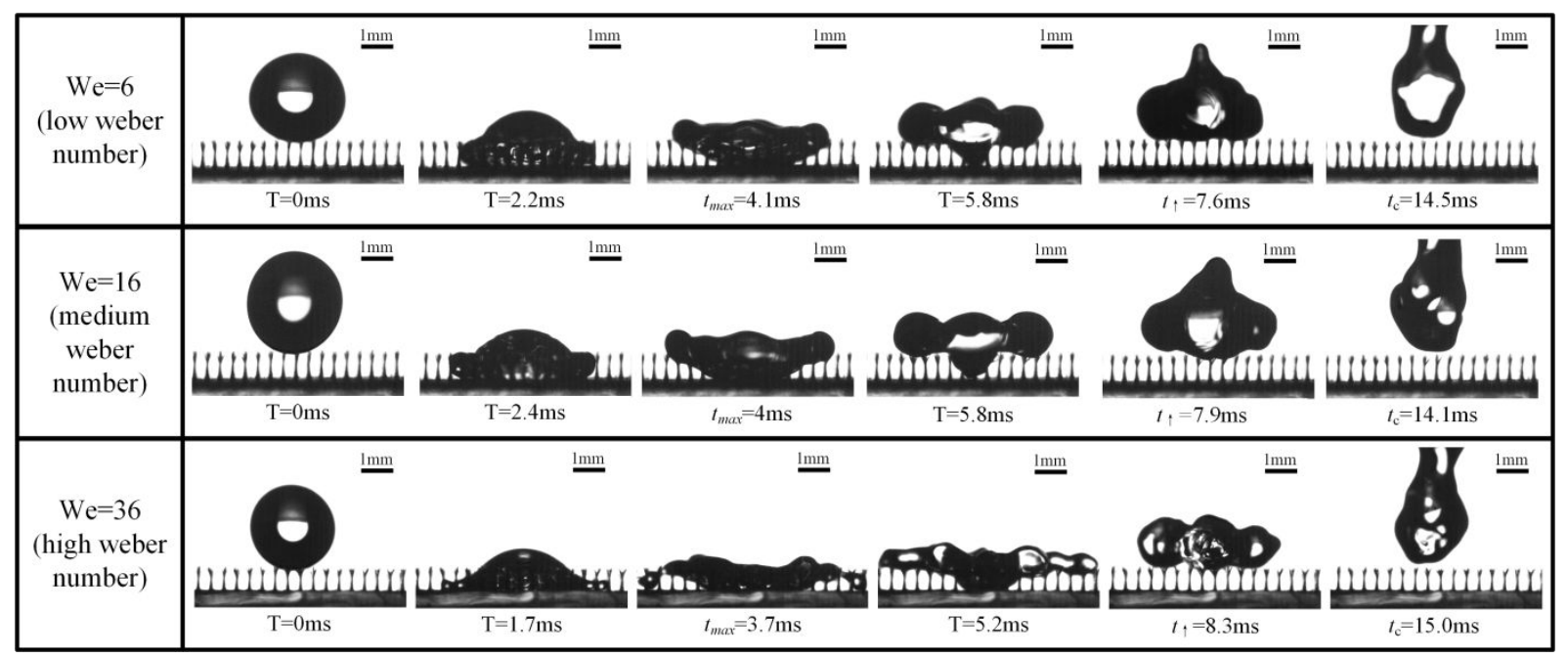

Figure S3-5. Under different Weber number $W e$, the bouncing states of a droplet on the SCAS with microstructural spacing ratio $S / D_{\mathrm{b}}=2.5$. The droplet radius is $R_{0}=1.45 \mathrm{~mm}$. 


\section{C3. The relationship between a droplet's size and its rebound states}

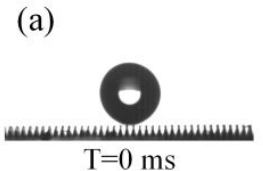

(b)

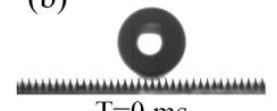

(c)

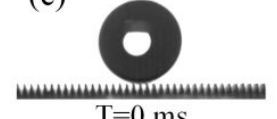

(d)

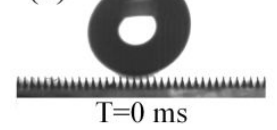

(e)

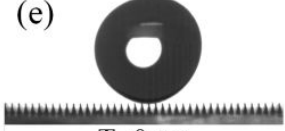

(f)

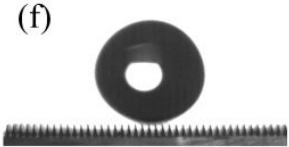

(g) $\mathrm{T}=0 \mathrm{~ms}$
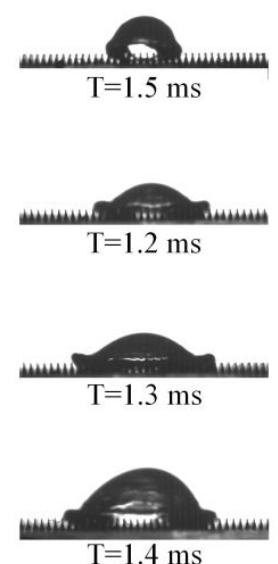
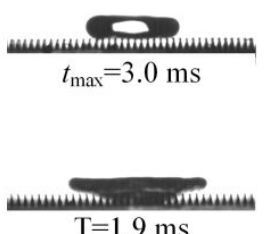

$\mathrm{T}=1.9 \mathrm{~ms}$

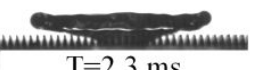

$\mathrm{T}=2.3 \mathrm{~ms}$

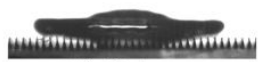

$\mathrm{T}=2.4 \mathrm{~ms}$
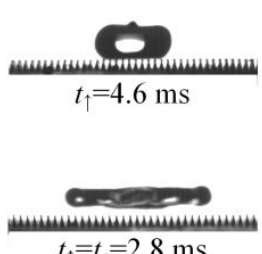
$t_{\uparrow}=t_{\mathrm{c}}=2.8 \mathrm{~ms}$

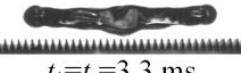
$t_{\uparrow}=t_{\mathrm{c}}=3.3 \mathrm{~ms}$

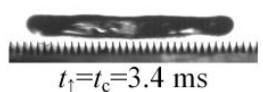
$t_{\uparrow}=t_{\mathrm{c}}=3.4 \mathrm{~ms}$
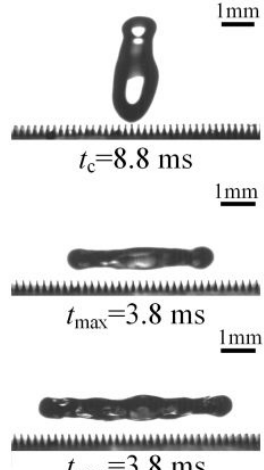
$t_{\max }=3.8 \mathrm{~ms}$

$\underline{1 \mathrm{~mm}}$
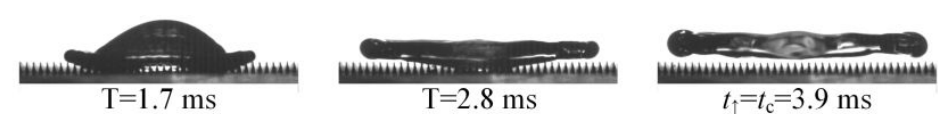

$t_{\uparrow}=t_{\mathrm{c}}=3.9 \mathrm{~ms}$
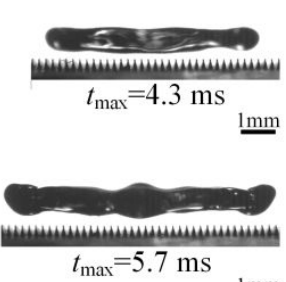
$t_{\max }=5.7 \mathrm{~ms}$
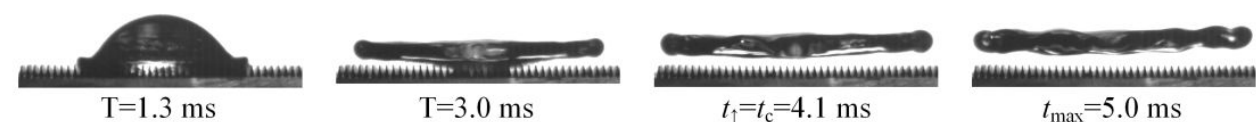
$t_{\uparrow}=t_{\mathrm{c}}=4.1 \mathrm{~ms}$

$t_{\max }=5.0 \mathrm{~ms}$

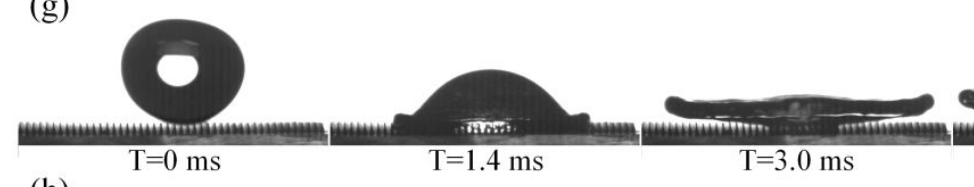

(h)
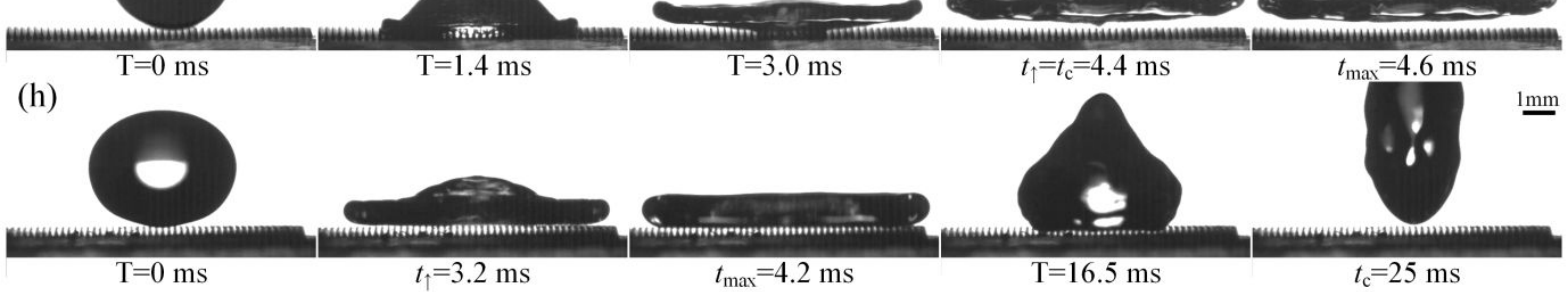

Figure S4. A snapshot picture of the moment when the droplets of different volumes (a) $V=3 \mu \mathrm{L}$, (b) $V=5 \mu \mathrm{L}$, (c) $V=8 \mu \mathrm{L}$, (d) $V=12 \mu \mathrm{L}$, (e) $V=15.5 \mu \mathrm{L}$, (f) $V=28 \mu \mathrm{L}$, (g) $V=50 \mu \mathrm{L}$, (h) $V=75 \mu \mathrm{L}$, rebound on the SCAS with $S / D_{\mathrm{b}}=1$ at $W e \approx 20$. 
(a)

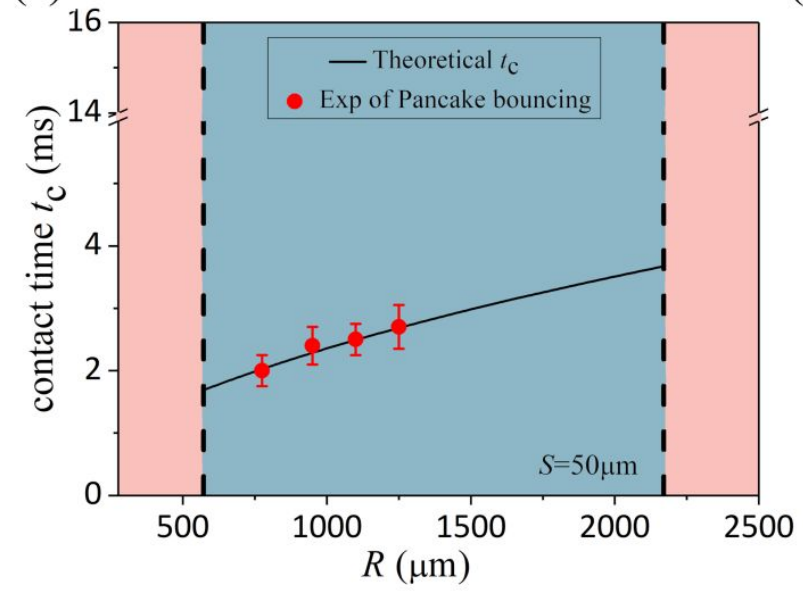

(b)

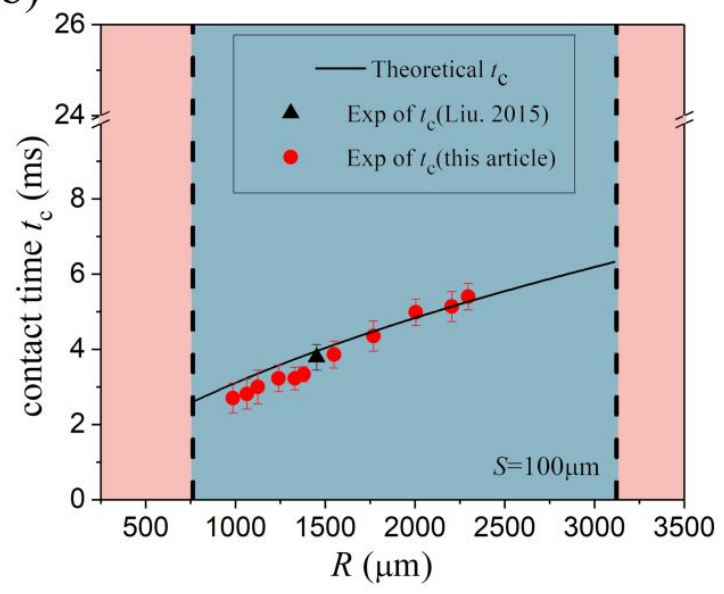

(c)

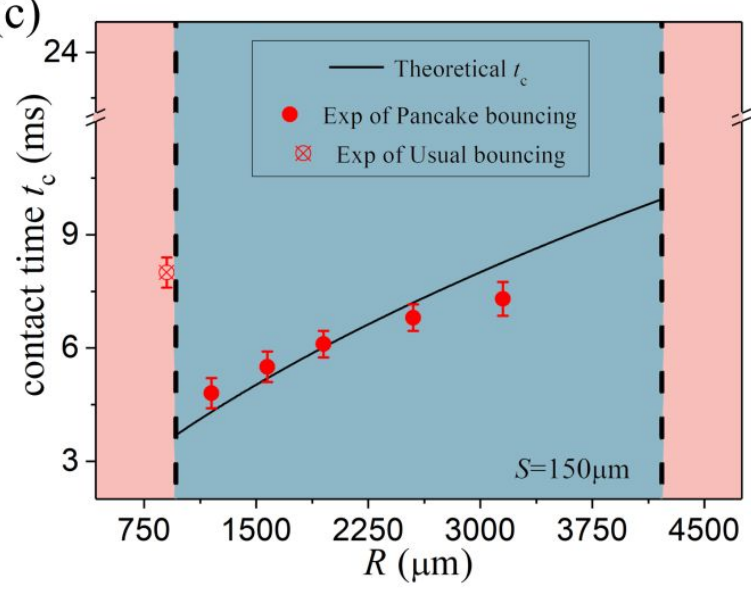

Figure S5. The relationship between the contact time $t_{\mathrm{c}}$ and radius $R$ of droplets under different microstructural spacing: (a) $S=50 \mu \mathrm{m}$, (b) $S=100 \mu \mathrm{m}$ and (c) $S=150 \mu \mathrm{m}$. The red part in the figure corresponds to the area where the droplets cannot theoretically undergo pancake bouncing with the ratio of the nanosphere structures $b / a=1$ at $W e \approx 20$. 


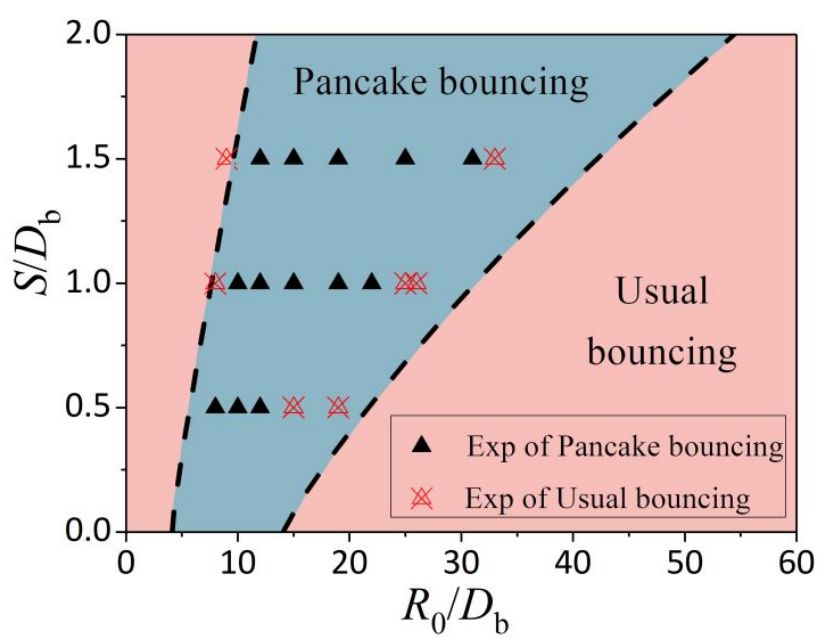

Figure S6. A phase diagram showing the pancake bouncing of droplets with different droplet radiuses and microstructure sizes. The ratio of the nanosphere structures $b / a=1$ at $W e \approx 20$. The dotted lines represent the comparable timescales $k$ for pancake bouncing. The experimental points on the right side have errors in fitting with the theory. The reason for this error is that the size of rebounded droplets has a great influence on the effective mass parameter $f_{\mathrm{t}}$, which makes the timescale $k$ cannot be well fitted.

\section{C4. The relationship between the spacing of the conical arrays $S / D_{\mathrm{b}}$ and half apex angle $\alpha$}

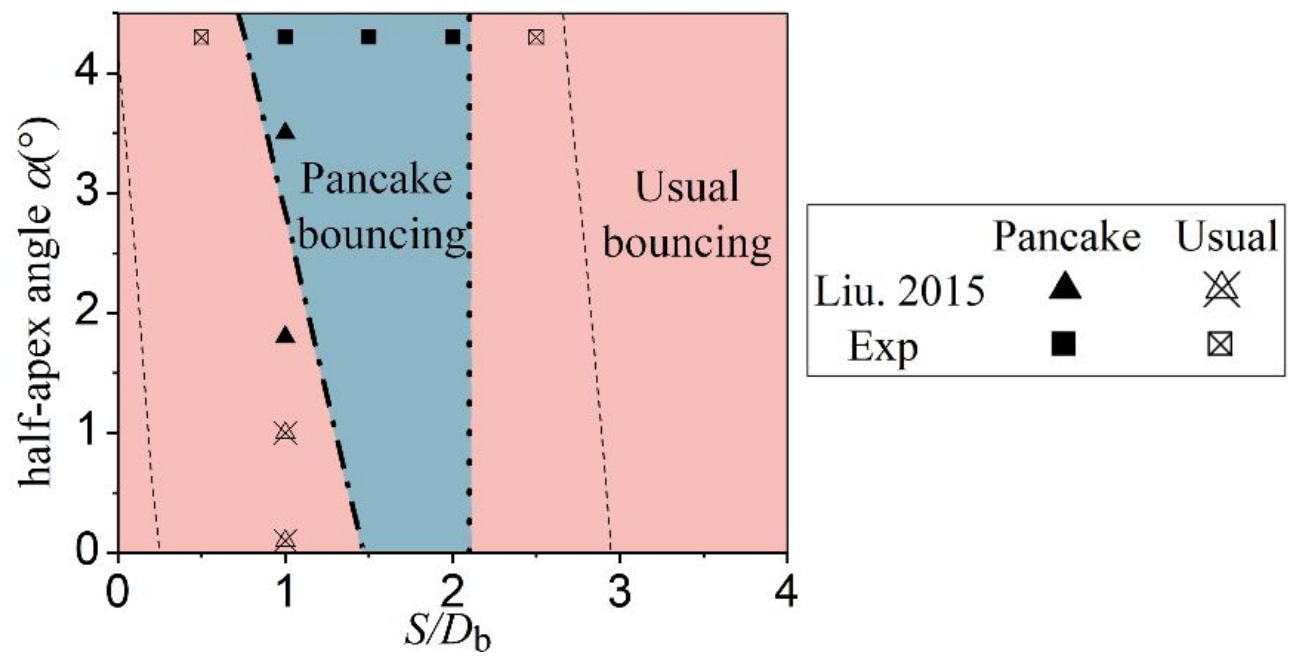


Figure S7. A phase diagram showing the range of bouncing states of a droplet $\left(R_{0}=1.45 \mathrm{~mm}\right)$ rebounded under different ratios between the spacing of the conical arrays $S / D_{\mathrm{b}}$ and half apex angle $\alpha$ under the limitations of the pancake bouncing. The ratio of the nanosphere structures $b / a=1$ and $W e \approx 16$. The pointed line represents the appropriate wetting states $S / D_{\mathrm{b}} \leq 2.2$ when $b / a=1$, as shown in Figure 2(a); the dash dotted line represents the energy criterion (based on Equation (7)); and the dashed lines represent the comparable timescales $k$ for pancake bouncing, respectively.

\section{REFERENCES}

(1) Wu, H. P.; Zhu, K.; Wu, B. B.; Lou, J.; Zhang, Z.; Chai, G. Z. Influence of Structured Sidewalls on the Wetting States and Superhydrophobic Stability of Surfaces with Dual-scale Roughness. Appl. Surf. Sci. 2016, 382, 111-120.

(2) Chen, Z.; Tian, F.; Hu, A.; Li, M. A facile process for preparing superhydrophobic nickel films with stearic acid. Surf. Coat. Technol. 2013, 231, 88-92.

(3) Liu, Y. H.; Mhyman, G.; Bormashenko, E.; Hao, C. L.; Wang, Z. K. Controlling Drop Bouncing Using Surfaces with Gradient Features. Appl. Phys. Lett. 2015, 107, 051604.

(4) Liu, Y. H.; Moevius, L.; Xu, X. P.; Qian, T. Z.; Yeomans, J. M.; Wang, Z. K. Pancake Bouncing on Superhydrophobic Surfaces. Nat. Phys. 2014, 10, 515-519. 